\title{
Titan's surface inventory of organic materials estimated from Cassini RADAR observations
}

\author{
Ralph D. Lorenz
}

\author{
Space Department, Johns Hopkins University Applied Physics Laboratory, USA \\ email: Ralph.lorenz@jhuapl.edu
}

\begin{abstract}
Cassini RADAR observations now permit an initial assessment of the inventory of two classes, presumed to be organic, of Titan surface materials: polar lake liquids and equatorial dune sands. Several hundred lakes or seas have been observed, of which dozens are each estimated to contain more hydrocarbon liquid than the entire known oil and gas reserves on Earth. Dark dunes cover some $20 \%$ of Titan's surface, and comprise a volume of material several hundred times larger than Earth's coal reserves. Overall, however, the identified surface inventories $\left(>3 \times 10^{4} \mathrm{~km}^{3}\right.$ of liquid, and $>2 \times 10^{5} \mathrm{~km}^{3}$ of dune sands) are small compared with estimated photochemical production on Titan over the age of the Solar System. The sand volume is too large to be accounted for simply by erosion in observed river channels or ejecta from observed impact craters. The lakes are adequate in extent to buffer atmospheric methane against photolysis in the short term, but do not contain enough methane to sustain the atmosphere over geologic time. Thus, unless frequent resupply from the interior buffers this greenhouse gas at exactly the right rate, dramatic climate change on Titan is likely in its past, present and future.
\end{abstract}

\section{Discussion}

NitTleR: Does the similarity in the dune morphologies between Titan and the Earth imply similar grain size?

LORENZ: It does in the sense that there is an optimum size for the transport of granular materials in any given environment. If particles are very large, they have a low areato-mass ratio, so it is difficult for wind to pick them up against gravity. If they're very small, then they stick together effectively. If you play with sugar with different particle sizes you can see that the very fine grain stuff actually has some cohesion. As it turns out, the optimum particle size is more or less the same for Titan and the Earth. The wind speeds required to transport sandy material on Titan are quite low, maybe about a meter per second, which is comparable with what is expected for the tidal currents in the atmosphere created by Titan's eccentric orbit around Saturn. There are some outstanding issues with the dunes, and what they indicate about the prevailing wind direction, that challenges global circulation models right now. But there's a whole new field of aeolian geomorphology to be explored with Titan now and what it means for the meteorology.

SLOAN: Would you please define the word "smust" that appeared on one of your slides?

Lorenz: It's not a word I like; it wasn't even a paper I liked. Don (Donald) Hunten came up with this idea that the solid aerosol particles somehow absorb liquid ethane onto them and stop it from acting like a liquid. I can't remember the etymology of the term - I think it was a way to refer to something like "moist dust". You have to look it up - it was in Nature last year I believe. 
ZiURYS: You described some nice experiments where tholins and water lead to amino acids and pyrimidines and pyrroles, but how did you get rid of the nitrogen and produce sugars? Has anyone done experiments on that?

LOREnZ: To be honest, this whole story has not been explored in anywhere near the depth in the laboratory that it needs to be. I know Catherine Neish in Arizona is dabbling with some hydrolysis rate coefficients. But to really explore the complexity here - and what stuff is removed and what stuff precipitates out - somebody needs to do that. I can't imagine these are particularly difficult experiments to at least have a stab at, but they may be difficult to do right.

ZiURYs: There may be some kind of reactions where you get $\mathrm{N}_{2}$ and that gets lost in the gas phase so you can make things without nitrogen ...

LORENZ: Right. There is the whole question of how these hydrolysis processes depend on the $\mathrm{pH}$ of the solution - whether you are hydrolyzing them with water, or perhaps, as is more likely in the Titan environment, a strong ammonia solution. Ammonia acts as an antifreeze in the water and brings the melting point down. There's a lot of stuff to explore.

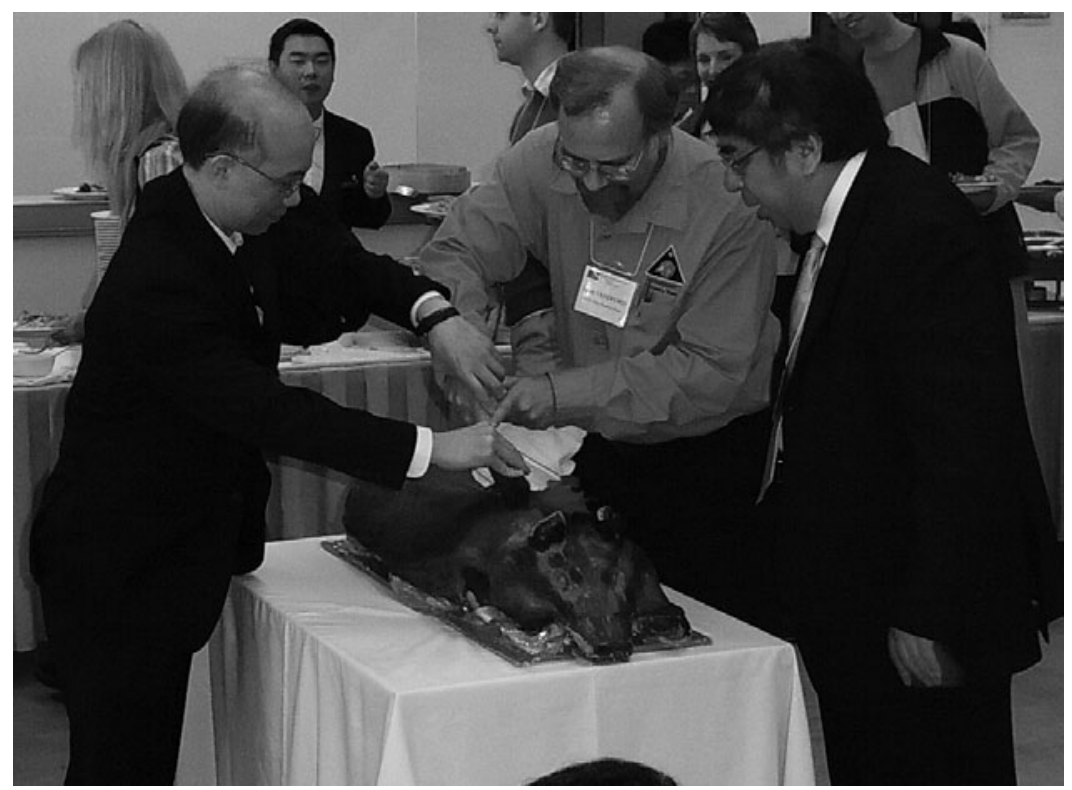

Scott Sandford performing the cutting of the pig ceremony, a Cantonese custom of ensuring the success at the opening of a major event. 